\title{
Application Study on Control Strategy of Underwater Platforms
}

\author{
Wei Zhang ${ }^{1, a}$, Qin-Guo Liu, ${ }^{1, b}$, Jun-Feng Pan ${ }^{1, c}$ and Rui Yang ${ }^{1, d,}$ \\ ${ }^{1}$ China Waterborne Transport Research Institute, Xitucheng $8{ }^{\#}$ Road, Haidian District, Beijing, \\ China \\ azhangwei@wti.ac.cn, b68861452@qq.com, '122040512@qq.com, dyangr@wti.ac.cn \\ ${ }^{*}$ Corresponding author
}

Keywords: Underwater Platforms, Control Strategy, Adjustment.

\begin{abstract}
This paper, focusing on control and adjustment of underwater platforms, aims to realize the efficient control strategy of underwater platform attitude and effectively improve the operation efficiency of underwater platforms.
\end{abstract}

\section{Introduction}

With the growing number of maritime transports and developments of marine resources, there are more and more maritime accidents. Necessary measures shall be taken as soon as possible to remove sunken ships and objects and reduce damages caused by them. In most cases, rescue and salvage works are carried out under the sea in harsh conditions, the position and attitude of under platforms may change at any time by ocean currents, thus produces great difficulties for underwater search and fixed-point rescue. The attitude and stability of underwater platforms are essential for fast and efficient underwater operation, so research on attitude control and adjustment of underwater platforms is needed to provide effective technical support for underwater rescue and salvage works.

\section{Underwater platform}

With part or most of human functions, underwater platform (underwater robot) is an automatic control assembly which can do some works for human in the marine environment. Generally, it makes three dimensional motions under the water on power provided by cables or propeller mounted on its energy source drive and equipment for lighting and image taking, sonar and other observation equipment, various sensors and professional tools for underwater works can be mounted on it.

The underwater platform in this research adopts streamline shape; the pressure shell, a cylindrical shell with hemispherical head, has two horizontal propellers on both sides and two vertical propellers on the upper part with buoyancy materials and a stand on the lower part. The cameras and other devices can be placed in the pressure shell.

The structure schematic diagram of underwater platform is shown in Figure 1. 


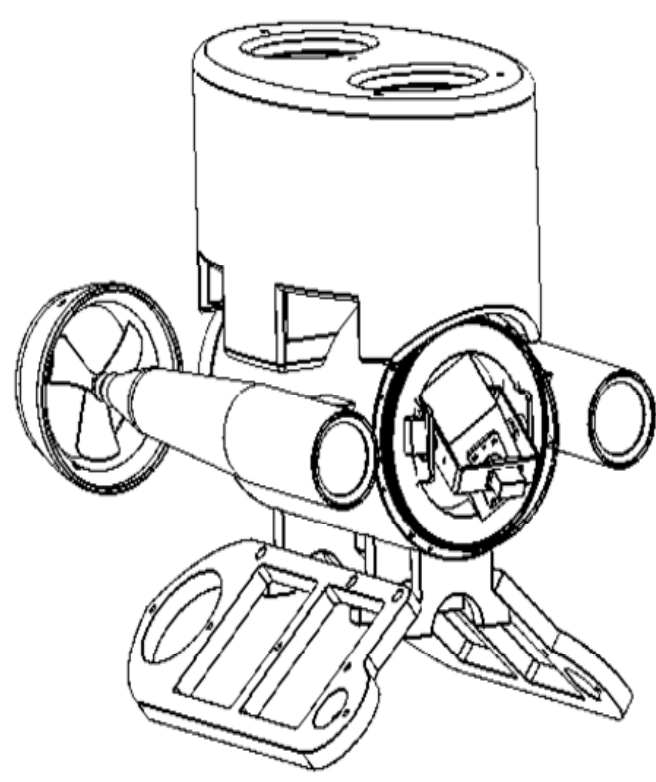

Figure 1 Structure Schematic Diagram of Underwater Platform

\section{Motion control method of underwater platform}

In order to make the underwater platform work normally and complete the established task, there must be a good motion control method for the basic motion control system. The directional movement and depth-fixed movement of the underwater platform is achieved by controlling the propeller, the control method of propeller mainly includes:

1.Kinematics and dynamics models are the basis for controlling the underwater platform, so first discuss and work out the kinematics and dynamics models of underwater platform;

2.Analyze and discuss the basic control loop of the direction control of underwater platform and develop direction control measures based on the control loop;

3.Analyze and discuss the basic control loop of the depth control of underwater platform and develop depth control measures based on the control loop;

4.Take the underwater platform, whose actuator is the propeller, as the study object, analyze and discuss the basic control loop of underwater platform station keeping combined with direction and depth control loop and control measures and develop measures based on the control loop.

\section{Direction control}

The function of direction control is to make the underwater platform move in target course. The advantages and drawbacks of the direction control measures and algorithm of underwater platform is one of the deciding factors which influence the control effect. The difference, achieved by the control system of the direction control, between the real course output, measured by the underwater platform with sensor, and the target course is the current course error, i.e.

$$
e_{\psi}=\psi_{i}-\psi_{o}(1)
$$

Where:

$$
e_{\psi} \text {-current course error; } \psi_{i} \text { - target course; } \psi_{o} \text { - real course measured by sensor }
$$

The control algorithm selected in the control system will calculate the controlled quantity according to the course error and control the signal outputting by thrust allocation logic and produce 
new course. By the continuous cycle of control in the program, the error ${ }^{e_{\psi}}$ will be reduced to 0 and the direction control of underwater platform is realized.

Data collected by electronic compass for course control is the data between $0^{\circ}$ and $360^{\circ}$, so when measuring the course angle of underwater platform by optical fibre gyro, data used here shall also be transformed to the data between $0^{\circ}$ and $360^{\circ}$. There will be a sudden change in the data when it is $0^{\circ}$ or $360^{\circ}$, thus brings inconvenience for the course control of underwater platform, if do not deal with the data, the underwater platform will move in situ and lose control.

The direction control measure is achieved by the following 4 steps:

(1) Calculate the error. Calculate the current course error ${ }^{e_{\psi}}$ based on formula (1) and update it in every control cycle.

(2) Deal with the error $e_{\psi}$. Deal with the error when its value is beyond the range of $\left(-180^{\circ}\right.$, $180^{\circ}$ ). If $e_{\psi}>180$, subtract $180^{\circ}$ from it, if $e_{\psi<-180}$, add $180^{\circ}$ to it, then restart step 2 ; when $e_{\psi}$ is within the range of $\left(-180^{\circ}, 180^{\circ}\right)$, take $e_{\psi}$ into the control algorithm.

(3) Control the algorithm calculation and output. Take the $e_{\psi}$ dealt in step 2 into the control algorithm and, finally output the data by sensor after thrust allocation logic.

(4) Verify the validity of direction control. If the control system still makes direction control, return to step 1 to continue the direction control cycle, if the control system trends to end the direction control, quit the direction control cycle. The aim of this step added in direction control is to make convenience for the control system to end the direction control at any time, if it's a big control cycle outside, the control system can start the direction control at any time and realize any start and stop function of direction control.

\section{Depth control}

The function of depth control is to make the underwater platform move in target depth. The depth control of underwater platform is similar to direction control, so influence to other degrees of freedom is the main consideration. The difference, achieved by the control system of the depth control, between the real depth, measured by the underwater platform with sensor (generally is depth gauge), and the target depth is the current depth error, i.e.

$$
e_{d}=d_{i}-d_{o}
$$

Where:

$$
e_{d} \text { - current depth error; }{ }_{i} \text { - target depth; }{ }^{d} \text { - real depth measured by sensor }
$$

Depth control algorithm converts the current depth error to controlled quantity and converts the influence to other degrees of freedom to depth control to regulated quantity by the corresponding control algorithm, take thrust allocation by thrust allocation logic combined with the influence to other degrees of freedom, control signals will be created to control the underwater platform and a new depth output will be achieved. By the continuous cycle of control in the program, the error $e_{d}$ will be reduced to 0 and the depth control of underwater platform is realized. 
Direction control takes no consideration of the influence to other degrees of freedom;In contrast, the difficulty of depth control is that it may influence other degrees of freedom during the depth control, such as trim, roll and so on. The specific degrees of freedom which may be influenced are related with the propeller configuration of underwater platform or the whole composition of underwater platform equipment as well as the environmental factors. The whole component of control measures is easier than that of direction control, but the thrust allocation logic in control is rather complex, which is related with the propeller configuration of underwater platform, dynamics of underwater platform and other factors.

The depth control measure mainly includes:

(1) Calculate the error ${ }^{e_{d}}$. Calculate the current depth error based on formula (2).

(2) Take the depth error $e_{d}$ into the control algorithm and create the control signal and output it after thrust allocation

(3) Verify whether continue the depth control. The concrete content and function are the same with step 4 of direction control measure.

\section{Study on control strategy}

Take water tank experiment by underwater platform and collect the kinematics data of underwater platform by the experiment. Build a kinematics simple model of underwater platform based on experimental data analysis and process. A simulation research can be carried out by using the simple model in order to find an optimal method for attitude control of underwater platform.

Analyze and process the experimental data and build the course (direction) kinematic model and depth (depth) kinematic model of underwater platform by MATLAB system identification toolbox.

\section{Conclusion}

The practical application in attitude control and adjustment of underwater platform is discussed with experimental data and simulation study after repeated underwaterexperiments and data tests, thus full combination between theoretical research and experimental research is realized. After repeated and effective experimental measurements, the control strategies provided in this paper can realize a good control of the course and depth of underwater platform and the research result and technical experience achieved here provide good technology solutions for underwater platform to adjust its attitude quickly and accurately and keep a steady state in rescue and salvage works.

\section{Acknowledgement}

This research was financially supported by the WTI Foundation.

\section{References}

[1] JiaXianjun. Position and Attitude Control of Small Underwater Rescue Robot and its Application in Underwater Research and Rescue [D] . Zhejiang university, 2014.

[2] XUYuru, LIPengchao. The development trend of underwater robot [ J ] . The Journal Nature, 2011, 33 ( 3 ):125-131.

[3] Zhu Jing etc. Fuzzy Control Principle and Application [M] . Beijing: China Machine Press, 1995. 